\title{
Achieving consistent multiple daily low-dose Bacillus anthracis spore inhalation exposures in the rabbit model
} \author{
Alison E. Director-Myska ${ }^{3}$, Tonya L. Nichols ${ }^{4}$ and Sarah C. Taft ${ }^{4}$ \\ ${ }^{1}$ Battelle Biomedical Research Center, Battelle Memorial Institute, Columbus, OH, USA \\ ${ }^{2}$ Department of Defense, Naval Surface Warfare Center, Dahlgren, VA, USA \\ ${ }^{3}$ Department of Defense, Defense Threat Reduction Agency, Fort Belvoir, VA, USA \\ ${ }^{4}$ National Homeland Security Research Center, U.S. Environmental Protection Agency, Cincinnati, OH, USA
}

Roy E. Barnewall ${ }^{1}$, Jason E. Comer ${ }^{1}$, Brian D. Miller ${ }^{1}$, Bradford W. Gutting ${ }^{2}$, Daniel N. Wolfe ${ }^{3}$,

\section{Edited by:}

Margaret Louise Mansel Pitt, United

States Army Medical Research

Institute of Infectious Diseases, USA

Reviewed by:

Mark Estes, University of Georgia, USA

Michael L. Vasil, University of

Colorado Medical School, USA

\section{*Correspondence:}

Sarah C. Taft, National Homeland

Security Research Center, U.S.

Environmental Protection Agency, 26

West Martin Luther King Drive, MS

NG-16, Cincinnati, OH 45268, USA.

e-mail: taft.sarah@epa.gov

Repeated low-level exposures to biological agents could occur before or after the remediation of an environmental release. This is especially true for persistent agents such as $B$. anthracis spores, the causative agent of anthrax. Studies were conducted to examine aerosol methods needed for consistent daily low aerosol concentrations to deliver a lowdose (less than $10^{6}$ colony forming units (CFU) of $B$. anthracis spores) and included a pilot feasibility characterization study, acute exposure study, and a multiple 15 day exposure study. This manuscript focuses on the state-of-the-science aerosol methodologies used to generate and aerosolize consistent daily low aerosol concentrations and resultant low inhalation doses to rabbits. The pilot feasibility characterization study determined that the aerosol system was consistent and capable of producing very low aerosol concentrations. In the acute, single day exposure experiment, targeted inhaled doses of $1 \times 10^{2}, 1 \times 10^{3}$, $1 \times 10^{4}$, and $1 \times 10^{5} \mathrm{CFU}$ were used. In the multiple daily exposure experiment, rabbits were exposed multiple days to targeted inhaled doses of $1 \times 10^{2}, 1 \times 10^{3}$, and $1 \times 10^{4} \mathrm{CFU}$. In all studies, targeted inhaled doses remained consistent from rabbit-to-rabbit and dayto-day. The aerosol system produced aerosolized spores within the optimal mass median aerodynamic diameter particle size range to reach deep lung alveoli. Consistency of the inhaled dose was aided by monitoring and recording respiratory parameters during the exposure with real-time plethysmography. Overall, the presented results show that the animal aerosol system was stable and highly reproducible between different studies and over multiple exposure days.

Keywords: Bacillus anthracis, inhalation exposures, low-dose, subchronic exposures, spores, anthrax, aerosol system

\section{INTRODUCTION}

Risk-based site-specific decision making for biothreat agents such as Bacillus anthracis poses unique challenges because of the adverse combination of high persistence and high lethality. B. anthracis is a Gram-positive, rod-shaped, facultative anaerobic, spore-forming bacterium that is very stable in the environment (Inglesby et al., 1999, 2002). Each potential B. anthracis exposure route (gastrointestinal, cutaneous, and inhalation) manifests itself in different clinical symptoms, with inhalational anthrax being the most lethal (Dixon et al., 1999). The B. anthracis Ames spore size ranges from 1.14 to $2.27 \mu \mathrm{m}$ in length (mean $1.52 \pm 0.19$ ) and $0.7-1.0 \mu \mathrm{m}$ in diameter (mean 0.81 \pm 0.06 ; Carrera et al., 2007) making it the ideal size for inhaling deep into the human alveolar spaces (Schlesinger, 1985; Hinds, 1999). Inhalation exposure can result from spores that aerosolize upon the initial release as well as subsequent re-aerosolization after settling on surfaces (Weis et al., 2002). Given the high lethality of $B$. anthracis spores from the inhalation route of exposure, the risk evaluation of contaminated sites requires the ability to model the inhalation hazard posed by spores that may remain on surfaces prior to or subsequent to decontamination.
Successful inhalation aerosol exposure studies targeting lowdoses and the effects of multiple doses are needed to improve the understanding of the potential risks associated with low levels of inhaled spores. The principles used when generating, sizing, quantifying, and sampling aerosols of inert materials also hold true for bioaerosols, i.e., for aerosolizing materials of biological origin. In light of this, the principles and techniques used for bioaerosols, including highly infectious and lethal bacteria like B. anthracis, have changed little in the last 60 years (Henderson, 1952; May, 1973; Stephenson et al., 1988; Cox and Wathes, 1995; Reponen et al., 1997; Roy and Pitt, 2006). One of the major limitations encountered when sampling infectious bioaerosols for dose determination is that the method must account for the viability of the organism. Multiple techniques are available to quantify microorganisms after sample collection such as direct microscopic count, turbidity measurements, polymerase chain reaction, gravimetric weight measurement, and culture. However, for spore-forming microorganisms such as B. anthracis, the culture method is used to distinguish viable from non-viable cells.

Limited low-dose-response data for B. anthracis continues to challenge the development of effective risk-based approaches 
for potential contamination events (Wilkening, 2006; Gutting et al., 2008). With some exceptions (Druett et al., 1953), historical B. anthracis inhalation exposure studies with published dose-response data relied on high doses that were associated with multiple times the median lethal doses $\left(\mathrm{LD}_{50}\right)$ to ensure lethality in control animals (Galloway et al., 2004; Oscherwitz et al., 2010; Yee et al., 2010). Earlier studies were conducted with a variety of test species (e.g., guinea pigs, rabbits, various non-human primate species) and exposure parameters such as differing B. anthracis strains, varying routes of exposure, and numerous aerosol particle sizes (Fellows et al., 2001; Lawrence et al., 2009; Levy et al., 2011). However, very few published animal exposure studies include adequate dose ranges for low-dose extrapolation and sufficient descriptions of exposure products to allow for dosimetric adjustments.

In view of the limited low-dose exposure data and the critical need to support risk-based site-specific decision making, exposure studies targeting low-doses were initiated in the rabbit model. A pilot characterization study was first conducted to determine whether it was feasible to deliver low-doses of $B$. anthracis Ames strain spores via the inhalation route in the animal aerosol system. After achieving consistent results in the pilot study, both an acute exposure study and multiple daily low-dose inhalation exposure study were conducted. This manuscript details the complex aerosol design and techniques utilized to achieve the required consistent low-doses on multiple exposure days.

\section{MATERIALS AND METHODS CHALLENGE MATERIAL}

The B. anthracis Ames strain spores were prepared and characterized at Battelle (Columbus, $\mathrm{OH}, \mathrm{USA}$ ) and were stored at $4-8^{\circ} \mathrm{C}$ in sterile water (Thermo Scientific HyClone, Logan, UT, USA) with $1.0 \%$ phenol (Sigma-Aldrich, St. Louis, MO, USA). Prior to use, the spores were washed with endotoxin-free water four times and were diluted to the appropriate concentration in endotoxinfree sterile water and $0.01 \%$ Tween 20 . The spore suspensions contained less that $5 \%$ vegetative cells and debris and over $95 \%$ were refractile under examination with phase contrast microscopy (Leica Microsystems, Wetzlar, Germany). Prior to aerosolization, the spores were enumerated (as described in Sample Analysis) and diluted to the proper concentration required to yield the targeted dose. The final concentrations of the nebulizer samples were targeted to be $1 \times 10^{4}, 1 \times 10^{5}, 1 \times 10^{6}$, and $1 \times 10^{7}$ colony forming units $(\mathrm{CFU}) / \mathrm{mL}$ depending on the study.

\section{INHALATION SYSTEM}

The inhalation system used is similar in design and operation to those used at the U.S. Army Medical Research Institute of Infectious Diseases (USAMRIID; Hartings and Roy, 2004; Roy and Pitt, 2006). The aerosol system was operated within a self-contained Class III biological safety cabinet (BSC III) as diagrammed in Figure 1.

\section{Aerosol generation subsystem}

Air was supplied to the system by an in-house air system filtered through two high efficiency particulate air (HEPA) capsule filters (Pall Corp, Port Washington, NY, USA) and a carbon filter (Pall
Corp, Port Washington, NY, USA). The air was split into dilution airflow of approximately $8.6 \mathrm{~L} / \mathrm{min}$ and a nebulizer/bypass airflow of approximately $7.5 \mathrm{~L} / \mathrm{min}$, maintained by mass flow controllers (MFC, Sierra, Monterey, CA, USA). The MFC were calibrated prior to use. The dilution air was humidified via a bubbler as needed to maintain humidity within a desired range of $53-83 \%$ as required per standard operating procedure for the aerosol system. A modified Microbiological Research Establishment type threejet Collison nebulizer (BGI, Waltham, MA, USA) with a precious fluid jar (BGI, Waltham, MA, USA) was used to generate a controlled delivery of aerosolized $B$. anthracis spores, from a liquid suspension. Collison nebulizers are designed to generate aerosols having an approximate mean diameter of $1-2 \mu \mathrm{m}$. The nebulizer was characterized and flow verified for a pressure that results in an approximately $7.5 \mathrm{~L} / \mathrm{min}$ flow, which normally is approximately $172,375-248,220 \mathrm{~Pa}$ (or $25.0-36.0$ pounds per square inch), Collison nebulizer dependant.

\section{Delivery subsystem}

After the agent aerosol was generated by the Collison nebulizer, it exited the Collison and traveled down a $3.75-\mathrm{cm}$ diameter, $40 \mathrm{~cm}$ long stainless steel cylinder (mixing tube) that mixed the aerosol with dilution air. The aerosol then entered the top of the exposure chamber through another cylinder with a tapered $14 \mathrm{~cm}$ long slit on each side. The total airflow entering the exposure chamber was approximately $16 \mathrm{~L} / \mathrm{min}$. The aerosol entered the chamber through these slits to fill the exposure chamber, washed over the exposure target (muzzle or head), and was then exhausted out of the exposure chamber through another cylinder at the bottom that contained slots on two sides, each $19.5 \mathrm{~cm}$ in length. The aerosol was pulled through the chamber using a vacuum pump (GAST, Benton Harbor, MI, USA) that maintained a slight negative pressure (from -0.25 to -0.01 inches of water) within the exposure chamber, as measured using a differential pressure gage (Magnehelic, Dwyer Instruments, Inc., Michigan City, IN, USA). The exhaust aerosol was filtered by two HEPA cartridge filters (Pall Corp, Port Washington, NY, USA) before exiting the system into the BSC III.

\section{Exposure chamber}

The exposure chamber was a plexiglass box with internal dimensions of approximately $20.5 \mathrm{~cm} \times 20.5 \mathrm{~cm} \times 40 \mathrm{~cm}$ (Length $\times$ Width $\times$ Height). A port approximately $15 \mathrm{~cm}$ in diameter was located on one side of the chamber where an animal's head or muzzle entered into the exposure chamber. Rubber dental dam was stretched across the opening and held in place with an o-ring gasket. The animal's muzzle was pushed through a small hole in the dental dam, producing a seal to prevent leakage around the opening prior to aerosol generation. Four additional ports were located in the chamber; two $1 / 4$ inch diameter ports for collection of aerosol samples [one for enumeration of spores and one for aerosol particle sizing with an Aerodynamic Particle Sizer (APS) spectrometer (Model 3321; TSI, Inc., Shoreview, MN, USA)], one 3/4 inch diameter port to measure temperature and humidity (Temperature Stick, Testo, Sparta, NJ, USA) and one 1/4 inch diameter port to measure the differential pressure within the exposure chamber in relation to the surrounding atmosphere within the BSC III. 


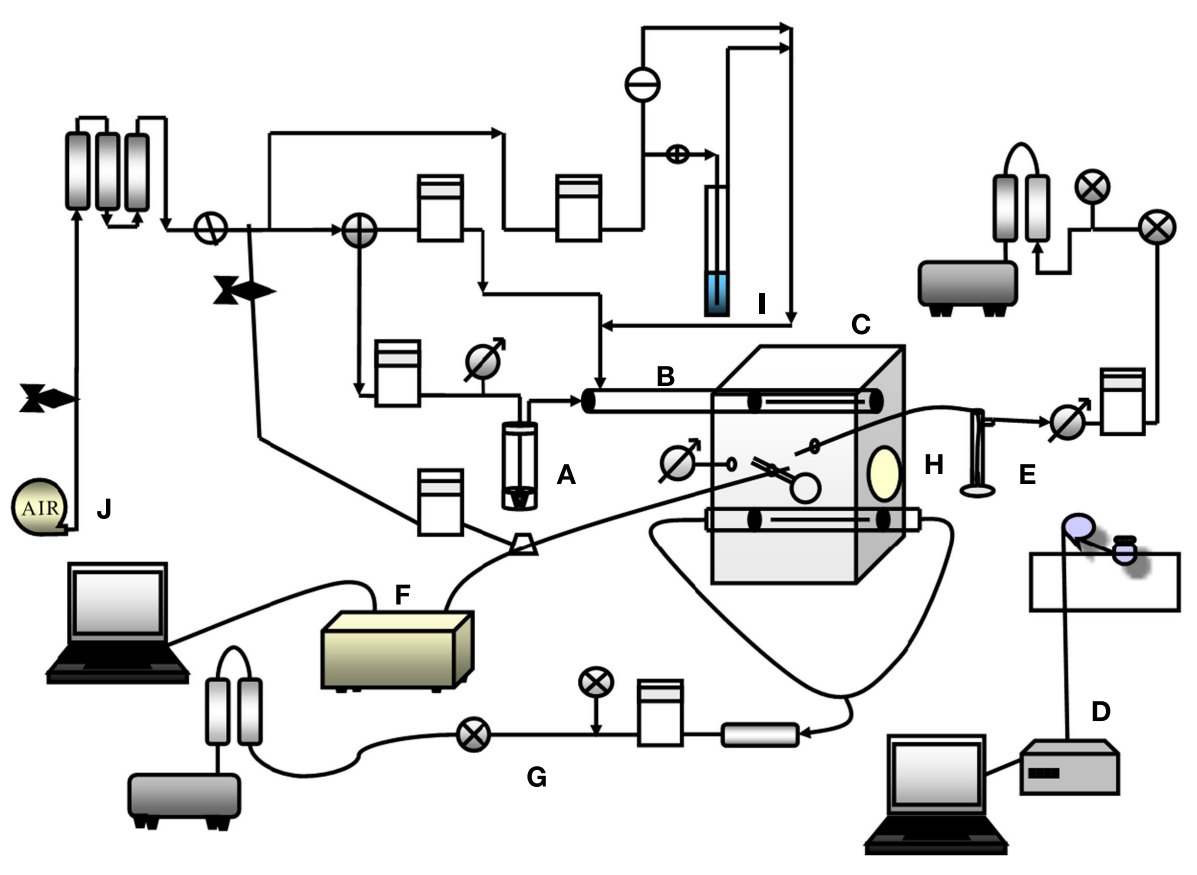

FIGURE 1 | Diagram of the inhalation exposure system used throughout the testing. Diagram shows all subsystems. A is the aerosol generator, Collison nebulizer, $\mathbf{B}$ is the delivery and mixing tube, $\mathbf{C}$ is the exposure chamber along with ports for the animal muzzle temperature and humidity probe, APS and impinger sampling ports and differential pressure gage port, $\mathbf{D}$ is the plethysmography system, $\mathbf{E}$ is the Impinger, $\mathbf{F}$ is the APS particle sizing system, $\mathbf{G}$ is the exhaust, $\mathbf{H}$ is the exposure port, $\mathbf{I}$ is the humidifier/bubbler, and $\mathbf{J}$ is the air supply.
Thus, the sampling from the impinger and APS spectrometer, and exposure of the animal all occur from the same chamber. Temperature and humidity were monitored once at approximately $5 \mathrm{~min}$ into the aerosol generation during each test.

\section{Sampling/monitoring}

Aerosol concentration and aerosol particle size distribution were determined by analysis of atmospheric samples drawn from the exposure chamber. The atmospheric samples were collected in an impinger (Model 7541, Ace Glass Inc.) filled with approximately $20 \mathrm{~mL}$ of sterile water that sampled at approximately $6.0 \pm 0.3 \mathrm{~L} / \mathrm{min}$. The sampling rate was achieved by maintaining a vacuum of $\geq 60,955 \mathrm{~Pa}$ (or $\geq 18^{\prime \prime}$ mercury; $\mathrm{Hg}$ ) across the exhaust connection of the impinger to maintain the flow from the impinger critical orifice and the sampling flow rate was monitored with a mass flow meter (MFM, Sierra, Monterey, CA, USA). The MFM was calibrated prior to use. Each impinger was verified to operate properly prior to use using a mini-Buck calibrator ${ }^{\circledR}$ flow meter (A.P. Buck Inc., Orlando, FL, USA). A new sterile impinger was used for each test day. The liquid in the impinger was diluted and enumerated by the spread plate technique to quantify CFU/mL. Filters $\left(0.45 \mu \mathrm{m}\right.$, Nalgene ${ }^{\circledR}$, Catalog no. 145-0045; Fisher Scientific, Pittsburgh, PA, USA) were also used to quantify CFU/mL when the spore concentration in the impinger was anticipated to be very low (for the 100 and 1,000 inhaled dose targets; see Sample Analysis). Enumeration results, along with the volume of liquid in the impinger, sampling rate, and sampling duration, were used in the calculation of the aerosol concentration expressed as CFU/L of air.
The aerosol particle size was determined once at approximately 5 min into the aerosol generation during each test using an APS spectrometer, which draws an atmospheric sample from the exposure chamber at $0.25 \mathrm{~L} / \mathrm{min}$ with a diluter $(1.0 \mathrm{~L} / \mathrm{min}$ total sampling flow with $0.75 \mathrm{~L} / \mathrm{min}$ from the diluter and $0.25 \mathrm{~L} / \mathrm{min}$ from the exposure chamber). An APS was used because of its advantages over other methods. These advantages include near real-time data measurements, aerodynamic diameter measurements, ease of instrument operation, and the generation of electronic data that is easy to process and export to a report.

\section{Plethysmography}

Body plethysmography was performed real-time on each animal during agent challenge to measure respiratory parameters in the acute exposure and multiple daily exposure studies. These parameters, which included tidal volume (TV), total accumulated TV (TATV), and minute volume (MV), were calculated from the measured volumetric displacement of air caused by the movement of the thoracic cavity of the animal while it was in a sealed chamber called a plethysmograph. The data generated for each animal was used to determine the TATV, which along with the aerosol concentration was used in calculating the inhaled dose.

Prior to animal exposures, the plethysmography was calibrated to establish unit (baseline) and air volume displacements from 5 to $40 \mathrm{~mL}$ to simulate animal respiration. Rabbits were secured within a plethysmography restraint device with the head protruding out of a port that was sealed with rubber dental dam and held into place with two plexiglass guillotines. The plethysmograph was connected to a pneumotach (Hans Rudolph, Inc., Kansas City, 
MO, USA) that was attached to a differential pressure transducer (Model DP-45; Validyne Engineering Corp., North Ridge, CA, USA). Pressure differential measurements from inhalations and exhalations were transmitted to Biosystems XA version 1.5.7 software (Biosystems XA, Buxco Electronics, Sharon, CT, USA) which then calculated and recorded respiratory function.

\section{SAMPLE ANALYSIS}

Impinger samples were enumerated by the serial dilution $\left(10^{-1}\right.$ to $10^{-3}$ ) and plating $0.1 \mathrm{~mL}$ of each dilution on tryptic soy agar (TSA) plates (Hardy Diagnostics, Santa Maria, CA, USA) in quintuplicate. Diluted samples were mixed via vortexing in a capped vial prior to subsequent dilutions. At each different dilution, $0.1 \mathrm{~mL}$ was spread onto each of five TSA plates, which were placed in a secondary container and incubated for $24-72 \mathrm{~h}$ at $37 \pm 2^{\circ} \mathrm{C}$, and then enumerated. Impinger samples from the $1 \times 10^{2}$ and $1 \times 10^{3}$-CFU targeted inhaled doses were enumerated by filtering $1.0 \mathrm{~mL}$ of the impinger volume, overlaying the filter on a TSA agar plate, performed in quintuplicate and examining the growth on the filter. Briefly, $1.0 \mathrm{~mL}$ of the sample was passed through a sterile $0.45 \mu \mathrm{m}$ filter (Nalgene ${ }^{\circledR}$ ) analytical test filter funnel (Catalog no. 145-0045; Fisher Scientific, Pittsburgh, PA, USA). The filter then was placed face up on top of a TSA plate, incubated for $24-72 \mathrm{~h}$ at $37 \pm 2{ }^{\circ} \mathrm{C}$, and then enumerated. After the incubation period, the plates were enumerated to determine the average number of colonies on the replicate plates. The impinger sample concentration was determined using Eq. 1:

$$
\begin{aligned}
C_{\mathrm{i}} & =\frac{(A \times \mathrm{DF})}{0.1 \mathrm{~mL}} \\
& C_{\mathrm{i}}=\text { Impinger sample concentration }(\mathrm{CFU} / \mathrm{mL}) \\
& A=\text { Average CFU per plate }(\mathrm{CFU}) \\
& \mathrm{DF}=\text { Dilution factor (unitless). }
\end{aligned}
$$

\section{INHALED DOSE CALCULATION}

The total inhaled dose $\left(D_{\text {inh }}\right)$ was calculated from the impinger sample concentration $\left(C_{\mathrm{i}}\right)$, impinger sample volume $\left(V_{\mathrm{i}}\right)$, impinger sampling rate $(S)$, exposure duration $(T)$, and total inhaled TV ( $V_{\text {inh }}$ ) according to the Eq. 2 below. The total number of viable spores captured during each exposure was the product of the impinger sample concentration $\left(C_{i}\right)$ and the impinger sample volume $\left(V_{\mathrm{i}}\right)$. The total number of CFUs was divided by the amount that was sampled through the impinger during the exposure time $(S \times T)$. The aerosol concentration $\left(C_{\mathrm{a}}\right)$ was $\left(C_{\mathrm{i}} \times V_{\mathrm{i}}\right)$ $(S \times T)^{-1}$. The total inhaled dose $\left(D_{\text {inh }}\right)$ was the product of the aerosol concentration multiplied $\left(C_{\mathrm{a}}\right)$ by the total inhaled TV $\left(V_{\text {inh }}\right)$ as detailed in Eq. 2:

$$
\begin{aligned}
& D_{\text {inh }}=\left[\left(C_{\mathrm{i}} \times V_{\mathrm{i}}\right)(S \times T)^{-1}\right] \times\left(V_{\mathrm{inh}}\right)=C_{\mathrm{a}} \times V_{\mathrm{inh}} \\
& D_{\text {inh }}=\text { Total inhaled dose }(\mathrm{CFU}) \\
& C_{\mathrm{i}}=\text { Impinger sample concentration }(\mathrm{CFU} / \mathrm{mL}) \\
& V_{\mathrm{i}}=\text { Impinger sample volume }(\mathrm{mL}) \\
& S=\text { Impinger sampling rate }(\mathrm{L} / \mathrm{min}) \\
& T=\text { Exposure duration }(\mathrm{min}) \\
& V_{\text {inh }}=\text { Total inhaled } \mathrm{TV}(\mathrm{L}), \text { as determined by plethysmogra- } \\
& \text { phy } \\
& \quad C_{\mathrm{a}}=\text { Aerosol concentration }(\mathrm{CFU} / \mathrm{L}) .
\end{aligned}
$$

\section{SPRAY FACTOR}

The pilot feasibility testing consisted of performing spray factor (SF) testing using low concentrations of B. anthracis spores in the aerosol generator (Collison Nebulizer). This was done to ensure the challenge system was capable of achieving the low-dose aerosols that would be required to achieve the low target doses in the subsequent rabbit studies. The SF is the ratio of the aerosol concentration to the starting concentration.

The SF can be used as a fundamental indicator to measure and assess the performance of an aerosol system. The SF is the ratio of the concentration of agent in the aerosol (aerosol concentration, $C_{\mathrm{a}}$ ) to the starting concentration used in the aerosol generator (nebulizer concentration, $C_{s}$; Hartings and Roy, 2004; Roy and Pitt, 2006). The aerosol concentration is the concentration obtained from sampling the aerosol, in this case with an impinger, and enumerating the impinger sample. The SF value is dependent on the configuration/design of the aerosol system and its operational parameters and the species or strain of the biothreat agent aerosolized (Hartings and Roy, 2004; Roy and Pitt, 2006). Any change to the aerosol system, operational parameters, or the biothreat agent could change the SF. The SF was calculated by dividing the aerosol concentration $\left(C_{\mathrm{i}} \times V_{\mathrm{i}}\right)(S \times T)^{-1}$ or $C_{\mathrm{a}}$ by the nebulizer concentration $\left(C_{s}\right)$, i.e., the starting nebulizer suspension concentration, and was used to predict aerosol concentration for a given starting nebulizer suspension concentration. During SF pilot testing the overall mean SF was determined for all the nebulizer concentrations examined. The formula for determining the SF is presented in Eq. 3:

$$
\begin{aligned}
& \mathrm{SF}=\frac{\left(C_{\mathrm{i}} \times V_{\mathrm{i}}\right)(S \times T)^{-1}}{C_{\mathrm{s}} \times 1000(\mathrm{~mL} / \mathrm{L})}=\frac{C_{\mathrm{a}}}{C_{\mathrm{s}}} \\
& \mathrm{SF}=\text { Spray factor } \\
& C_{\mathrm{i}}=\text { Impinger sample concentration }(\mathrm{CFU} / \mathrm{mL}) \\
& V_{\mathrm{i}}=\text { Impinger sample volume }(\mathrm{mL}) \\
& S=\text { Impinger sampling rate }(\mathrm{L} / \mathrm{min}) \\
& T=\text { Exposure duration }(\mathrm{min}) \\
& C_{\mathrm{a}}=\text { Aerosol concentration }(\mathrm{CFU} / \mathrm{L}) \\
& C_{\mathrm{s}}=\text { Nebulizer concentration }(\mathrm{CFU} / \mathrm{L}) .
\end{aligned}
$$

In the pilot feasibility SF study, the aerosol system performance characteristics were evaluated by aerosolizing four target nebulizer concentrations of $B$. anthracis spores multiple times over a 3-day period. The target nebulizer concentrations tested over the 3days were $1.0 \times 10^{4}, 1.0 \times 10^{5}, 1.0 \times 10^{6}$, and $1.0 \times 10^{7} \mathrm{CFU} / \mathrm{mL}$. Specifically, each concentration was tested a total of nine times (three times per day over 3 days). Each test consisted of a 10-min period of aerosol generation and sample collection. The SF values determined in pilot testing were used to plan the exposures in the acute and multiple daily exposure studies.

\section{AUTHORIZATION FOR THE USE OF EXPERIMENTAL ANIMALS}

All animal work was approved by and performed in accordance with Battelle's Institutional Animal Care and Use Committee (IACUC) and the US Army Medical Research and Materiel Command Animal Care and Use Review Office (ACURO). The studies were performed in a biosafety level 3 laboratory registered with the Centers of Disease Control and Prevention and inspected by the Department of Defense and the U.S. Department of Agriculture. 


\section{RESULTS \\ PILOT FEASIBILITY STUDY}

The pilot feasibility study performed SF testing to examine whether the inhalation system was capable of achieving the low target doses required for the subsequent rabbit studies. Target nebulizer concentrations of $1 \times 10^{4}, 1 \times 10^{5}, 1 \times 10^{6}$, and $1 \times 10^{7} \mathrm{CFU} / \mathrm{mL}$ were tested in triplicate each day over the 3 days. Quantification of the nebulizers indicated that the enumerated values were up to $30 \%$ lower from the target values. As a result, in an animal study the targeted inhaled doses may not be achieved if the desired or targeted nebulizer concentrations are not achieved with the resulting inhaled dose likely off target by a similar percentage as the nebulizer concentration. This is due to the fact that there is a direct linear relationship between the nebulizer concentration and the aerosol concentration. Table 1 shows the mean, standard deviation, and standard error for the SF for each test day, and for the complete testing with the SF value of $4.88 \times 10^{-7} \pm 2.88 \times 10^{-7}$. Overall, the pilot feasibility study confirmed that the targeted low-doses anticipated in the subsequent acute and multiple daily exposure studies were achievable with a relative standard deviation (RSD) of 63\%. Aerosol concentration (as measured via impinger sampling) strongly correlated with nebulizer concentration (data not shown). The remaining nebulizer suspensions when enumerated after aerosolization were found to not be statistically significantly higher than the nebulizer concentrations that had been targeted prior to aerosolization, which indicates that no loss of viable spores had occurred as a result of nebulization process. The data from the pilot feasibility study was used to plan the subsequent single and multiple challenge day rabbit studies required nebulizer concentrations and how much test atmosphere the rabbits needed to inhale to reach the desired doses.

Additional information was generated on the aerosol particle size (mass median aerodynamic diameter, MMAD) for each of the four nebulizer concentrations tested during the feasibility study. Using the APS spectrometer, the MMAD for the aerosol generated from each of the $B$. anthracis nebulizer suspensions at each

Table 1 | Pilot feasibility study mean spray factor data.

\begin{tabular}{llll}
\hline $\begin{array}{l}\text { Target nebulizer } \\
\text { concentration (CFU/mL) }\end{array}$ & \multicolumn{3}{c}{ Spray factor } \\
\cline { 2 - 4 } & Day 1 & Day 2 & Day 3 \\
\hline $1 \times 10^{4}$ & $4.62 \times 10^{-7}$ & $1.67 \times 10^{-7}$ & $9.32 \times 10^{-7}$ \\
$1 \times 10^{5}$ & $5.35 \times 10^{-7}$ & $4.20 \times 10^{-7}$ & $6.12 \times 10^{-7}$ \\
$1 \times 10^{6}$ & $4.22 \times 10^{-7}$ & $1.82 \times 10^{-7}$ & $6.37 \times 10^{-7}$ \\
$1 \times 10^{7}$ & $3.14 \times 10^{-7}$ & $1.50 \times 10^{-7}$ & $6.66 \times 10^{-7}$ \\
Mean & $4.33 \times 10^{-7}$ & $2.30 \times 10^{-7}$ & $7.11 \times 10^{-7}$ \\
Standard deviation & $1.40 \times 10^{-7}$ & $2.73 \times 10^{-7}$ & $2.06 \times 10^{-7}$ \\
Standard error & $4.05 \times 10^{-8}$ & $7.88 \times 10^{-8}$ & $5.95 \times 10^{-8}$ \\
Overall mean & & $4.58 \times 10^{-7}$ & \\
Overall $\%$ retate & &
\end{tabular}

Overall \% relative standard

63

deviation

Overall standard deviation

$2.88 \times 10^{-7}$

Overall standard error concentration varied from 0.83 to $1.06 \mu \mathrm{m}$ with geometric standard deviation (GSD) of 1.53 or lower (1.40-1.53). The MMAD values increased as the nebulizer concentration increased. This effect was most likely due to the fact that the APS spectrometer analyzes and sizes all particles sampled and counted by the instrument. The APS spectrometer does not distinguish between water droplets, debris particles, spores, etc. As a result, a greater proportion of small water droplet and debris were likely counted relative to spores at the lower $B$. anthracis spore concentrations aerosolized. As the spore concentration increased in the nebulizer, a greater proportion of $B$. anthracis spores were aerosolized and thus analyzed by the APS compared to water droplets and debris, thereby accounting for a larger percent of the total mass. The result was a shift to a larger measured particle size or MMAD. This effect occurred until a spore concentration was reached in the aerosol where the majority of particles counted and sized were only spores and the MMAD reflected predominately individual spores. Regardless, the aerosol particle sizes obtained during testing were particle size ranges that can reach the rabbit or human deep lung alveoli. The same trend phenomenon was seen with the aerosol particle sizes in the acute single day exposure and multiple daily exposure studies.

\section{ACUTE SINGLE DAY INHALATION EXPOSURE STUDY}

In the acute study, four groups of five rabbits were aerosol challenged to deliver targeted inhaled doses of $1 \times 10^{2}, 1 \times 10^{3}$, $1 \times 10^{4}$, and $1 \times 10^{5} \mathrm{CFU}$ of $B$. anthracis spores. Nebulizer concentrations used to achieve the desired doses were targeted to be $1 \times 10^{4}, 1 \times 10^{5}, 1 \times 10^{6}$, and $1 \times 10^{7} \mathrm{CFU} / \mathrm{mL}$ based off of the feasibility study SF testing. Plate counts of the impinger samples revealed that mean actual inhaled doses per group were $2.86 \times 10^{2}\left( \pm 4.32 \times 10^{1}\right), 2.06 \times 10^{2}\left( \pm 3.42 \times 10^{2}\right), 2.53 \times 10^{2}$ $\left( \pm 5.21 \times 10^{3}\right)$, to $2.75 \times 10^{5}\left( \pm 7.41 \times 10^{4}\right) \mathrm{CFU}$, which were slightly higher than the target inhaled doses. The primary causes for the higher actual dose delivered from the target dose were the starting nebulizer concentrations which were higher than anticipated, and the SF value, which was higher compared to what was determined in the pilot feasibility study. The mean SF and standard deviation was determined to be $1.26 \times 10^{-6} \pm 1.35 \times 10^{-7}$ (individual data not shown) with a standard error of $6.28 \times 10^{-8}$ and a RSD of $22 \%$. This mean SF is 2.7 times higher than the mean SF determined in the pilot feasibility study. The nebulizer concentrations were 1.37- to 1.96-fold higher than the targeted nebulizer concentrations. Figure 2 illustrates the group mean inhaled challenge doses for the test day. The figure illustrates the group mean inhaled dose (bars) and standard deviation against the targeted dose (dashed lines). Additionally, the figure shows that while the inhaled doses were slightly higher than target and the increase above the target was relatively consistent across the four doses. The mean MMAD for challenge material for each group as determined by an APS spectrometer readings varied from 0.82 to $1.12 \mu \mathrm{m}$ with GSD of 1.33 to 1.57 , and with a trend that the MMAD increased as the nebulizer concentration increased.

\section{MULTIPLE DAILY INHALATION EXPOSURE STUDY}

Three groups of seven rabbits were exposed to targeted inhaled doses of $1 \times 10^{2}, 1 \times 10^{3}$, and $1 \times 10^{4} \mathrm{CFU}$ during the multiple 


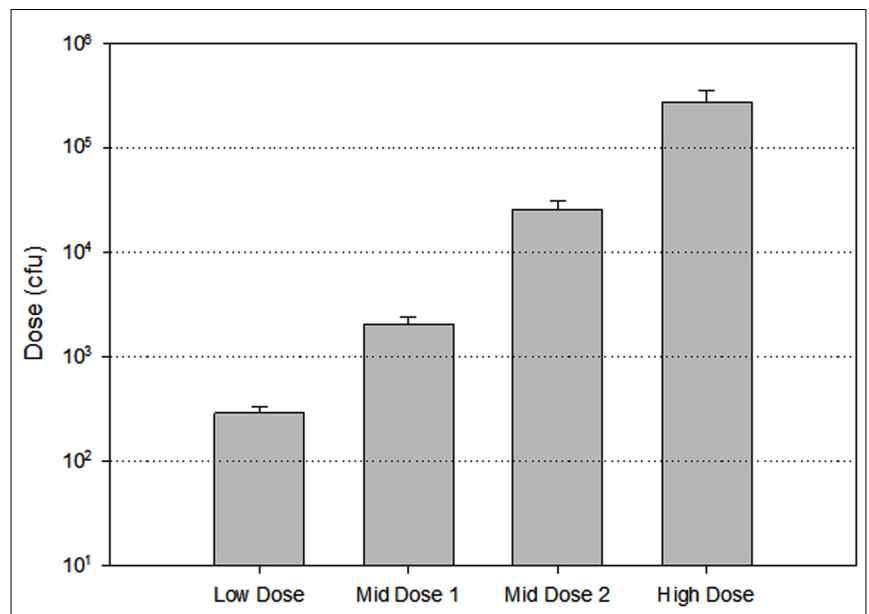

FIGURE 2 | Mean acute single day low-dose inhalation challenge data. The figure illustrates the mean inhaled dose (bars) with standard deviation compared to the target doses (dashed horizontal log increment lines). The low-dose target was $10^{2}$, the mid-dose 1 target was $10^{3}$, the mid-dose 2 target was $10^{4}$, and the high-dose target was $10^{5}$.

daily inhalation exposure study. Figure 3 illustrates the group mean challenge daily doses over the 15 challenge days. The figure illustrates the daily group mean inhaled dose (bars) and standard deviation against the targeted dose (dashed lines). The daily mean group inhaled dose values oscillate around the target dose. All challenge days had consistent dosing except Challenge Day 3 where the challenge dose of $1 \times 10^{2} \mathrm{CFU}$ was higher than expected. This increase was most likely caused by an error in the dilution of the challenge material. The actual mean doses for all 15 days were approximately 1.13-3.08 times the targeted inhaled dose and remained relatively consistent from animal to animal, which was very similar to the results obtained in the acute exposure study. Plate counts of the impinger samples revealed that individual mean actual inhaled doses of the 15-days of challenge ranged from $2.32 \times 10^{2}\left( \pm 1.28 \times 10^{1}\right)$ to $1.44 \times 10^{4}\left( \pm 5.99 \times 10^{3}\right)$ CFU. Similar to the acute exposure study results, the primary causes for the higher actual dose delivered from the target dose were the starting nebulizer concentrations, which were higher than anticipated, and the SF value, which was higher compared to what was determined in the pilot feasibility study. The mean SF and standard deviation was determined to be $1.13 \times 10^{-6} \pm 5.28 \times 10^{-7}$ (individual data not shown and day 2 low-dose data excluded) with a standard error of $3.11 \times 10^{-8}$ and a RSD of $48 \%$. This mean SF is 2.5 times higher than the mean SF determined in the pilot feasibility study and similar to the SF results for the acute single day study. Although the starting nebulizer concentrations were higher than the target concentrations, it was determined that the volume of test atmosphere each rabbits was to inhale was not to be adjusted in order for method consistency with the acute single exposure study. The nebulizer concentrations were 1.15- to 1.32fold higher than the targeted nebulizer concentrations. The mean MMAD for challenge material for each group for all 15 days as determined by an APS spectrometer readings varied from 0.79 to $0.86 \mu \mathrm{m}$ and GSD of 1.43 to 1.61 , and with the trend that the mean

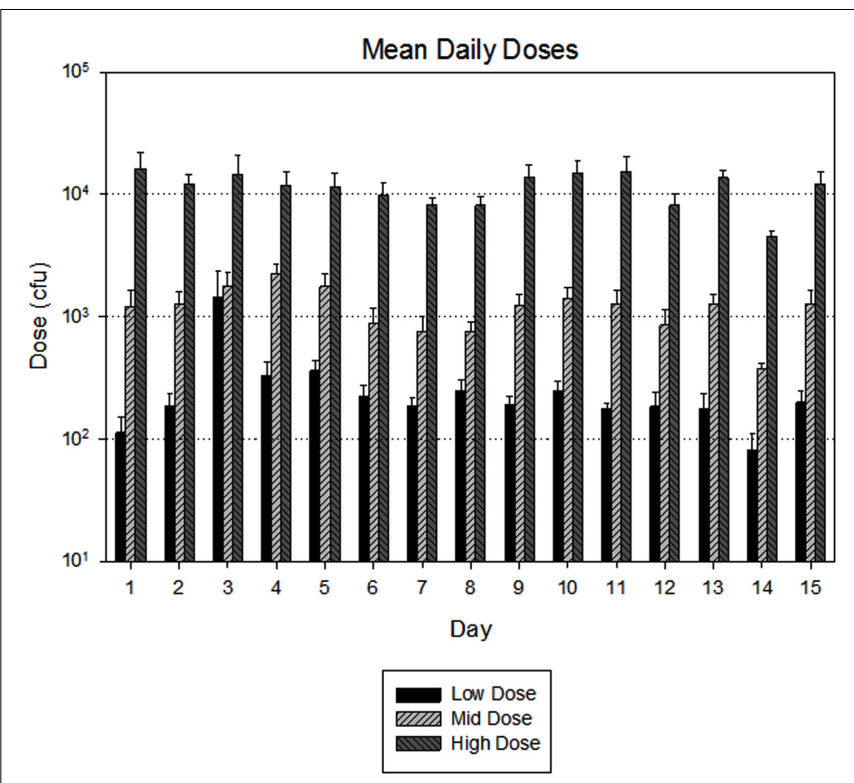

FIGURE 3 | Mean 15 day low-dose inhalation challenge data. The figure illustrates the mean inhaled dose (bars) with standard deviation compared to the target dose (dashed horizontal log increment lines). The low-dose target was $10^{2}$, the mid-dose target was $10^{3}$, and the high-dose target was $10^{4}$

group MMAD increased as the nebulizer concentration increased across all test days. Figure 4 is a log distribution plot of each group's mean MMAD and GSD along with a representative particle distribution graph from the APS spectrometer for the low-dose group.

\section{DISCUSSION}

These studies are the first known concerted effort to develop and deliver low-doses of $B$. anthracis spores via inhalation in a reproducible manner. The pilot feasibility study (see Table $\mathbf{1}$ for results) enabled the characterization of the aerosol system's ability to deliver low-doses which were confirmed in the acute and multiple daily inhalation exposure studies.

The pilot feasibility study determined a SF value when aerosolizing low concentrations of $B$. anthracis spores. The SF value is a unitless factor that represents the aerosol dilution that is to be expected during an aerosol experiment using the same biological agent in the same aerosol system (Roy and Pitt, 2006). In theory, the aerosol system should always generate the same mean SF or fall within the same range time for the material being aerosolized. Based on the SFs from the pilot feasibility study, it was determined that within an aerosol day the mean SF value could be \pm 2.3 times the target value and between days of \pm 5.6 times the target value which would cause the inhaled dose in an animal study to have the same variability. The RSD for the acute and 15-day study were 22 and $48 \%$, respectively, which both fall within the $63 \%$ that was determined in the pilot feasibility study.

One must also consider that the nebulizer concentration and impinger concentration used in determining the SF value and the 


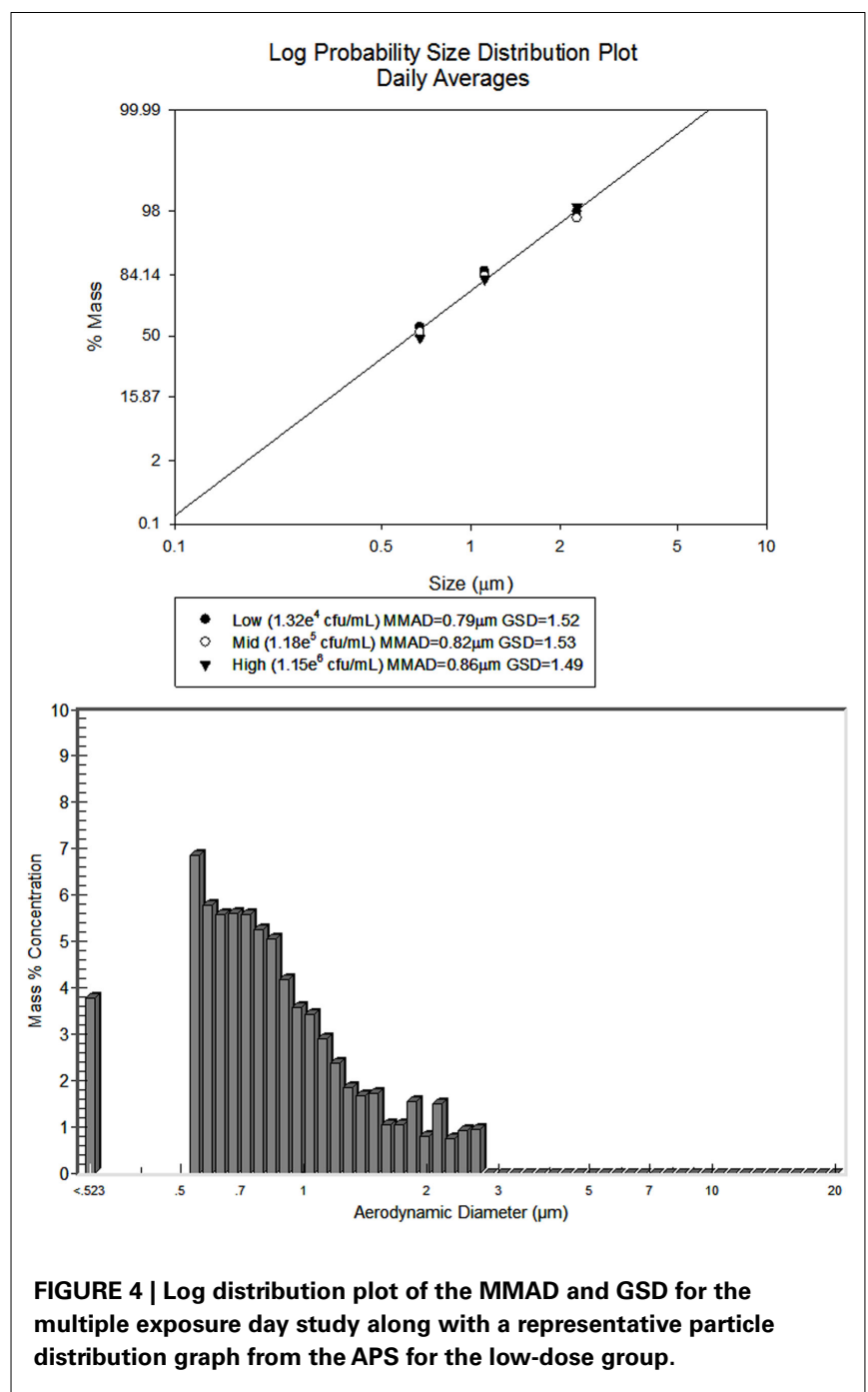

calculated dose delivered to an animal, respectively, are generated using the spread plate technique which has its own inherent variability. As a result, any problems such as a diluting error or plating the incorrect dilution, affects the nebulizer or impinger results, and will, in turn, affect the SF, and inhaled dose calculations.

In addition to the use of the SF as a tool to assess the performance of the aerosol system, the SF can be utilized in two other ways. First, the SF can be used to determine the nebulizer concentration $\left(C_{s}\right)$ needed to administer a specific total inhaled dose $\left(D_{\text {inh }}\right)$ over a fixed exposure time. For example, what nebulizer concentration $\left(C_{\mathrm{s}}\right)$ would be needed for a 10 -min exposure if the target total inhaled dose $\left(D_{\text {inh }}\right)$ were $1 \times 10^{6} \mathrm{CFU}$, and a historical SF of $1.0 \times 10^{-6}$ were used? If rabbits were aerosol challenged with $B$. anthracis spores for a 10-min exposure time $(T)$, and each rabbit's MV $\left(V_{\min }\right)$ were $1.0 \mathrm{~L}$ per minute, then $T \times V_{\min }$ $(10 \mathrm{~min} \times 1.0 \mathrm{~L} / \mathrm{min})$ would yield a total inhaled volume $\left(V_{\text {inh }}\right)$ of $10 \mathrm{~L}$. The necessary starting nebulizer concentration $\left(C_{\mathrm{s}}\right)$ based on the information to achieve the total inhaled dose $\left(D_{\mathrm{inh}}\right)$ is calculated as follows:

$$
\begin{aligned}
& V_{\text {inh }} \times C_{\mathrm{a}}=D_{\text {inh }} \\
& 10(\mathrm{~L}) \times C_{\mathrm{a}}=1 \times 10^{6}(\mathrm{CFU}) \\
& C_{\mathrm{a}}=1 \times 10^{6}(\mathrm{CFU}) / 10(\mathrm{~L})=1 \times 10^{5}(\mathrm{CFU} / \mathrm{L}) \\
& \mathrm{SF}=C_{\mathrm{a}} / C_{\mathrm{s}} \\
& 1 \times 10^{-6}=1 \times 10^{5}(\mathrm{CFU} / \mathrm{L}) \times C_{\mathrm{s}}^{-1} \\
& C_{\mathrm{s}}=1 \times 10^{5}(\mathrm{CFU} / \mathrm{L}) / 1 \times 10^{-6}=1 \times 10^{11}(\mathrm{CFU} / \mathrm{L}) \text { or } 1 \\
& \quad \times 10^{9}(\mathrm{CFU} / \mathrm{mL})
\end{aligned}
$$

Alternatively, with biological agents for which the concentration can be determined prior to the inhalation exposure, the SF can be used to determine the total TV $\left(V_{\text {inh }}\right)$ an animal must inhale to reach the target inhaled dose $\left(D_{\text {inh }}\right)$. In this scenario, the $\mathrm{MV}$ $\left(V_{\min }\right)$ of the animal is not known prior to challenge and is measured real-time during the exposure using plethysmography. As a consequence, all animals would inhale the same volume $\left(V_{\text {inh }}\right)$ of atmosphere and the exposure time $(T)$ would vary based in each animal's particular MV $\left(V_{\min }\right)$. For example, from a historical SF of $1.0 \times 10^{-6}$, the starting nebulizer concentration $\left(C_{s}\right)$ may be determined to be $1 \times 10^{9} \mathrm{CFU} / \mathrm{mL}$ prior to challenge to achieve the target inhaled dose $\left(D_{\text {inh }}\right)$ of $1 \times 10^{6} \mathrm{CFU}$. The necessary volume of aerosol to inhale $\left(V_{\text {inh }}\right)$ is calculated as follows. Based on the SF and starting concentration, the assumed aerosol concentration $\left(C_{\mathrm{a}}\right)$ will be:

$$
\begin{aligned}
C_{a} & =\mathrm{SF} \times C_{\mathrm{s}}=1 \times 10^{-6} \times\left(1 \times 10^{9} \mathrm{CFU} / \mathrm{mL}\right) \\
& =1 \times 10^{3} \mathrm{CFU} / \mathrm{mL} \text { or } 1 \times 10^{6} \mathrm{CFU} / \mathrm{L} \\
V_{\mathrm{inh}} & =D_{\mathrm{inh}} / C_{\mathrm{a}}=1 \times 10^{6}(\mathrm{CFU}) / 1 \times 10^{6}(\mathrm{CFU} / \mathrm{L})=1 \mathrm{~L}
\end{aligned}
$$

The advantage of conducting aerosol exposures using this second scenario compared to the first is that the precision and accuracy of achieving the target total inhaled dose is increased and the standard deviation of the mean of the total inhaled doses (mean $D_{\text {inh }}$ ) delivered to all the animals will be decreased. The primary cause for the larger standard deviation in first scenario is due to the inherent variability of the MV ( $\left.V_{\min }\right)$ among the animals. Thus, if the aerosol concentration is the same from exposure to exposure, and an animal with a $V_{\min }$ of $1 \mathrm{~L}$ per minute will receive/inhale twice the dose of an animal with a $V_{\min }$ of $0.5 \mathrm{~L}$ per minute. This effect is negated or reduced in the second scenario because all the animals are inhaling the same volume of aerosol but with different exposure times (Hartings and Roy, 2004). Several previous studies using this approach for designing $B$. anthracis exposure studies confirm this second approach is precise and accurate (Little et al., 2004; Klas et al., 2008; Migone et al., 2009).

These studies demonstrate how the total inhaled volume $\left(V_{\text {inh }}\right)$, as determined by plethysmography, can be used to calculate inhaled dose $\left(D_{\text {inh }}\right)$. One of the primary factors for the consistency of the inhaled dose delivered in these exposure studies was from the real-time monitoring and recording of respiratory parameters, in particular, $V_{\min }$ and $V_{\text {inh }}$ during the exposure, via plethysmography. As indicated previously (Hartings and Roy, 2004; Roy and Pitt, 2006), performing plethysmography real-time minimizes 
the variability resulting from individual rabbit's respiratory $V_{\min }$ compared to performing exposure of a defined time limit. Had a defined exposure time limit been used, the predicted dose received per animal and mean per dose group would have been approximately 30\% higher and with a larger standard deviation. Not all institutions perform real-time plethysmography and these studies are the first to use real-time plethysmography when performing low-dose exposures over multiple days, demonstrating its value to inhalation research.

Previous published data have used a similar descriptive term as SF called the aerosolization ratio. The aerosolization ratio is determined by dividing the total concentration of aerosolized organisms $\left(\mathrm{CFU} / \mathrm{m}^{3}\right)$ by the total concentration of organisms in the fluid (CFU/mL; Wang et al., 2004; Barth et al., 2009). When the total concentration of aerosolized organisms is converted to $\mathrm{L}$ in the aerosolization ratio $\left(\mathrm{m}^{3}=1000 \mathrm{~L}\right)$, the aerosolization ratio calculation is the same as the SF. The SF values generated in these studies cannot be compared to the aerosolization ratios since both the SF and aerosolization ration are dependent on the configuration/design of the aerosol system, its operational parameters, and the species/strain of the biothreat agent aerosolized.

The aerosol concentration can be altered (raised or lowered) by manipulations of the aerosol system. These manipulations include changing the aerosol generator, varying the output of the aerosol generator, varying the concentration of the material in the aerosol generator, and varying the dilution air. For the acute and multiple daily dose studies, it was most important to maintain consistency and to follow the same standard operating procedure in each study and on each study day. Therefore, adjusting the concentration of B. anthracis spores in the Collison nebulizer was selected as the most reliable procedure to ensure consistent results.

In the acute single day study, rabbits were exposed to targeted inhaled doses of $1 \times 10^{2}, 1 \times 10^{3}, 1 \times 10^{4}$, and $1 \times 10^{5} \mathrm{CFU}$ of $B$. anthracis spores. The actual doses were approximately 2.06-2.86 times the targeted inhaled dose and remained relatively consistent from animal to animal. In the multiple daily exposure study, rabbits were exposed to targeted inhaled doses of $1 \times 10^{2}, 1 \times 10^{3}$, and $1 \times 10^{4} \mathrm{CFU}$ of $B$. anthracis spores. The actual mean doses for all 15 days were approximately 1.13-3.08 times the targeted inhaled dose and remained relatively consistent from animal to animal,

\section{REFERENCES}

Barth, E. F., Reponen, T., and Succop, P. (2009). Evaluation of bioaerosol components, generation factors, and airborne transport associated with lime treatment of contaminated sediment. J. Air Waste Manage. Assoc. 59, 540-552.

Carrera, M., Zandomeni, R. O., Fitzgibbon, J., and Sagripanti, J. L. (2007). Difference between the spore sizes of Bacillus anthracis and other Bacillus species. J. Appl. Microbiol. 102, 303-312.

Cox, C. S., and Wathes, C. M. (1995). Bioaerosols Handbook. Boca Raton: CRC Press LLC.

which was very similar to the results obtained in the acute exposure study. The variability seen in the animal doses for both the acute single day study and 15 day study were within the predicted variability of the SF feasibility testing. One of the contributing factors for the higher than targeted doses the animals achieved was the higher than anticipated concentrations of spores in the Collison nebulizer. In both studies the nebulizer concentrations were 1.15-1.96 times higher than the target concentration. In spite of the higher nebulizer concentrations and the resultant higher doses delivered to the animals, the dose results presented in Figure 3 show that exposures produced by the aerosol system were stable and reproducible from day-to-day.

In all testing, the aerosol system produced aerosolized spores with MMAD particle sizes in all tests that were in a size range that can reach the rabbit deep lung alveoli. Additionally, it was demonstrated that the distribution of the aerosol was monodispersed as indicated by GSD less than 2.0. The biological effects of inhaled aerosols can be dependent upon the sites and degree of deposition within the respiratory tract. Further, the size and shape of inhaled aerosols is a critical factor in determining deposition mechanisms and the extent of penetration into the lung and alveolar regions. Aerosols with aerodynamic particle sizes between 1 and $5 \mu \mathrm{m}$ are suitable for inhalation studies in order to reach (Schlesinger, 1985; Hinds, 1999) and cause disease in the deep lung. Above this size, a larger portion of the aerosol is deposited in the upper respiratory tract (Schlesinger, 1985; Hinds, 1999). Large particles containing biological agents deposited in the upper respiratory tract may not cause disease, or may require a higher quantity (dosage) to cause disease or may cause only an upper respiratory disease. Therefore, if a study objective is to maximize deep lung deposition, then an aerosol with a size on the order of $1-5 \mu \mathrm{m}$ or lower is required; this range was attained throughout the three studies.

These studies have shown that with thorough characterization, traditional bioaerosol generation and collection techniques can be employed to achieve consistent and reproducible low-dose exposures within a day and between multiple test days in the animal model system. The data presented in this manuscript represent the first known study to show the repeatability of low-dose inhalation B. anthracis spore exposures.

Galloway, D., Liner, A., Legutki, J., Mateczun, A., Barnewall, R., and Estep, J. (2004). Genetic immunization against anthrax. Vaccine 22, 1604-1608.

Gutting, B. W., Channel, S. R., Berger, A. E, Gearhart, J. M., Andrews, G. A., Sherwood, R. L., and Nichols, T. L. (2008). Mathematically modeling inhalational anthrax. Microbe 3, 78-85.

Hartings, J. M., and Roy, C. J. (2004). The automated bioaerosol exposure system: preclinical platform development and a respiratory dosimetry application with nonhuman primates. J. Pharmacol. Toxicol. Methods $49,39-55$.
Henderson, D. W. (1952). An apparatus for the study of airborne infection. $J$. Hyg. (Lond.) 50, 53-68.

Hinds, W. C. (1999). Aerosol Technology: Properties, Behavior, and Measurement of Airborne Particles. New York: Wiley-Interscience.

Inglesby, T. V., Henderson, D. A., Bartlett, J. G., Ascher, M. S., Eitzen, E., Friedlander, A. M., Hauer, J., McDade, J., Osterholm, M. T., O’Toole, T., Parke, G., Perl, T. M., Russell, P. K., and Tonat, K. (1999). Anthrax as a biological weapon: medical and public health management. Working Group on Civilian Biodefense. JAMA 281, 1735-1745. 
Inglesby, T. V., Henderson, D. A., Bartlett, J. G., Ascher, M. S., Eitzen, E., Friedlander, A. M., Hauer, J., McDade, J., Osterholm, M. T., O’Toole, T., Parke, G., Perl, T. M., Russell, P. K., and Tonat, K. (2002). Anthrax as a biological weapon, 2002: updated medical and public health management. Working Group on Civilian Biodefense. JAMA 287, 2236-2252.

Klas, S. D., Petrie, C. R., Warwood, S. J., Williams, M. S., Olds, C. L., Stenz, J. P., Cheff, A. M., Hinchcliffe, M., Richardson, C., and Wimer, S. (2008). A single immunization with a dry powder anthrax vaccine protects rabbits against lethal aerosol challenge. Vaccine 26, 5494-5502.

Lawrence, W. S., Hardcastle, J. M., Brining, D. L., Weaver, L. E., Ponce, C., Whorton, E. B., and Peterson, J. W. (2009). The physiologic responses of Dutch belted rabbits infected with inhalational anthrax. Comp. Med. 59, 257-265.

Levy, H., Weiss, S., Altboum, Z., Schlomovitz, J., Rothschild, N., Blachinsky, E., and Kobiler, D. (2011). Lethal factor is not required for Bacillus anthracis virulence in guinea pigs. Microb. Pathog. 51, 345-351.

Little, S. F., Ivins, B. E., Fellows, P. F., Pitt, M. L., Norris, S. L., and Andrews, G. P. (2004). Defining a serological correlate of protection in rabbits for a recombinant anthrax vaccine. Vaccine 22, 422-430.
May, K. R. (1973). The Collison nebulizer. Description, performance and application. J. Aerosol Sci. 4, 235-243.

Migone, T. S., Subramanian, G. M., Zhong, J., Healey, L. M., Corey, A., Devalaraja, M., Lo, L., Ullrich, S., Zimmerman, J., Chen, A., Lewis, M., Meister, G., Gillum, K., Sanford, D., Mott, J., and Bolmer, S. D. (2009). Raxibacumab for the treatment of inhalational anthrax. N. Engl. J. Med. 361, 135-144. [Supplemental appendix].

Oscherwitz, J., Yu, F., and Cease, K. B. (2010). A synthetic peptide vaccine directed against the $2 ß 2-2 \beta 3$ loop domain 2 of protective antigen protects rabbits from inhalational anthrax. J. Immunol. 185, 3661-3668.

Reponen, T., Willeke, K., Ulevicius, V., Grinshpun, S. A., and Donelly, J. (1997). Techniques for dispersion of microorganisms into air. Aerosol Sci. Tech. 27, 405-421.

Roy, C. J., and Pitt, L. M. (2006). “Infectious disease aerobiology: aerosol challenge methods," in Biodefense: Research Methodology and Animal Models, ed. J. R. Swearengen (Boca Raton: CRC Press), 61-76.

Schlesinger, R. B. (1985). Comparative deposition of inhaled aerosols in experimental animals and humans: a review. J. Toxicol. Environ. Health 15, 197-214.
Stephenson, E. H., Moeller, R. B., York, C. G., and Young, H. W. (1988). Nose-only versus wholebody aerosol exposure for induction of upper respiratory infections of laboratory mice. Am. Ind. Hyg. Assoc. J. 49, 128-135.

Wang, H. X., Reponen, T., Adhikari, A., Willeke, K., and Grinshpun, S. A. (2004). Effect of fluid type and microbial properties on the aerosolization of microorganisms from metalworking fluids. Aerosol Sci. Technol. 38, 1139-1148

Weis, C. P., Intrepido, A. J., Miller, A. K., Cowin, P. G., Durno, M. A., Gebhardt, J. S., and Bull, R. (2002). Secondary aerosolization of viable Bacillus anthracis spores in a contaminated US senate office. JAMA 288 , 2853-2858.

Wilkening, D. A. (2006). Sverdlovsk revisited: modeling human inhalation anthrax. Proc. Natl. Acad. Sci. U.S.A. 103, 7589-7594.

Yee, S. B., Hatkin, J. M., Dyer, D. N. Orr, S. A., and Pitt, M. L. (2010). Aerosolized Bacillus anthracis infection in New Zealand white rabbits: natural history and intravenous levofloxacin treatment. Comp. Med. 60 , 461-468.

Disclaimer: The U.S. Environmental Protection Agency (EPA) through its Office of Research and Development co-funded and managed the research described herein under Interagency Agreement DW9792208901 with the Defense Technical Information Center, and in turn through Battelle Chemical, Biological, Radiological, and Nuclear Defense Information Analysis Center (CBRNIAC) Contract SP0-700-00-D-3180. The Department of Defense's Defense Threat Reduction Agency collaborated with EPA to fund the research herein under project numbers BA06TAS022 and CBS.PHYSIO.01.10.SW.005. No official endorsement should be inferred. EPA does not endorse the purchase or sale of any commercial products or services.

Received: 22 March 2012; paper pending published: 02 April 2012; accepted: 03 May 2012; published online: 13 June 2012.

Citation: Barnewall RE, Comer JE, Miller BD, Gutting BW, Wolfe DN, Director-Myska AE, Nichols TL and Taft SC (2012) Achieving consistent multiple daily low-dose Bacillus anthracis spore inhalation exposures in the rabbit model. Front. Cell. Inf. Microbio. 2:71. doi: 10.3389/fcimb.2012.00071

Copyright (C) 2012 Barnewall, Comer, Miller, Gutting, Wolfe, Director-Myska Nichols and Taft. This is an open-access article distributed under the terms of the Creative Commons Attribution Non Commercial License, which permits noncommercial use, distribution, and reproduction in other forums, provided the original authors and source are credited. 